\title{
O impacto do acesso aberto na produção e difusão de conhecimento sobre a Covid-19
}

\author{
The impact of open access on the production and dissemination of \\ knowledge about Covid-19
}

\author{
Tatiana Bermúdez-Rodrígueza,* (C) \\ Vinicius Muraroa (D) \\ Ana Carolina Spattia (i)
}

Cristina Abreu Sampaio Leme Monaco ${ }^{\mathrm{a}, \mathrm{b}}$ (D)

\begin{abstract}
RESUMO: A corrida científica ocasionada pelo surto do novo coronavírus (SARS-CoV-2) tem posto em evidência a relevância do compartilhamento de dados e resultados de pesquisa de forma mais rápida e aberta do que a do atual sistema de comunicação científica, bem como o papel das mídias sociais nesse contexto. O objetivo deste artigo é analisar o impacto do acesso aberto (open access) na produção e difusão do conhecimento sobre a COVID-19 nos meios tradicionais de comunicação científica e na web social. Via indicadores bibliométricos e altmétricos, analisamos um conjunto de 6.631 artigos e revisões das bases PubMed e Scopus, publicados entre janeiro e abril de 2020. Os resultados evidenciaram que 0 volume das publicações científicas relacionadas à COVID-19 aumentou a uma taxa média mensal de 166\% no período analisado, com destaque às publicações na modalidade acesso aberto. Os países com maior número de publicações são aqueles que tiveram mais casos registrados da COVID-19. No geral, observouse maior atenção da mídia social e redes de cooperação mais intensas nas publicações científicas de acesso aberto se comparadas às de acesso restrito. Ao combinar indicadores bibliométricos e altmétricos, o artigo permitiu não somente caracterizar a evolução e difusão da produção científica sobre a COVID-19, mas compreender a relação entre a pandemia provocada pelo novo coronavírus e a interação da sociedade em torno dos produtos de pesquisa. Nossos resultados reforçam a importância de se implementar práticas de acesso aberto para criar e/ou fortalecer redes de colaboração de pesquisa e estimular publicações sobre temas emergentes e de interesse mundial.
\end{abstract}

Palavras-chave: COVID-19; Indicadores Bibliométricos; Indicadores Altmétricos; Acesso Aberto; Redes de cooperação.

ABSTRACT: The race to publish results during the outbreak of the new coronavirus (SARS-CoV-2) has highlighted the relevance of sharing data and research results more quickly and openly than the current scientific communication system, as well as the role of social media in this context. This paper aims to analyze the impact of open access on the production and dissemination of knowledge about COVID-19 in traditional scientific communication and on the social web. We analyzed 6,631 papers and reviews of PubMed and Scopus databases, published between January and April 2020, through bibliometric and altmetric indicators. The results showed that the volume of scientific open access publications related to COVID-19 increased by an average monthly rate of $166 \%$ in the period analyzed. The countries with the highest number of publications are those that had the most registered cases of COVID-19. In general, the scientific open access publications had greater attention from social media and more intense cooperation networks than compared to non-open access publications. The combination of bibliometric and altmetric indicators allowed us not only to characterize the evolution and diffusion of scientific production on COVID-19 but also to understand the relationship between the pandemic caused by the new coronavirus and the interaction of society around research products. Our findings reinforce the importance of open access practices to create and strengthen research collaboration networks and to stimulate publications on emerging themes of global interest.

Keywords: COVID-19; Bibliometric Indicators; Altmetric Indicators; Open Access; Cooperation Networks.

a Departamento de Política Científica e Tecnológica, Instituto de Geociências, Universidade Estadual de Campinas, Campinas, SP, Brasil.

b Secretaria da Educação do Estado de São Paulo, Campinas, SP, Brasil.

*Correspondência para/Correspondence to: Tatiana Bermúdez-Rodríguez. E-mail: ladytb@unicamp.br. Endereço institucional: Rua Carlos Gomes, 250 - Cidade Universitária, Campinas - SP, 13083-855.

Recebido em/Received: 21/08/2020; Aprovado em/Approved: 07/10/2020.

Artigo publicado em acesso aberto sob licença CC BY 4.0 Internacional (c)( 


\section{INTRODUÇÃO}

Em março de 2020, a COVID-19 (sigla para Coronavirus Disease 2019) foi declarada uma pandemia pela Organização Mundial da Saúde (OMS) (WHO et al., 2020). A causa da doença foi um novo tipo de coronavírus (da família Coronaviridae), identificado então pela sigla SARS-CoV-2 devido à Síndrome Respiratória Aguda Grave ocasionada também por anteriores do gênero. Com efeito, desde janeiro de 2020, tem crescido a um ritmo bastante acelerado o número de trabalhos relacionados à doença, configurando uma corrida científica sem precedentes (TORRES-SALINAS et al., 2020).

O desafio imposto aos cientistas, especialmente no início de uma epidemia, é identificar resultados relevantes de pesquisa em um intervalo de tempo ainda curto (GORBALENYA, 2020), quando a disponibilidade de dados epidemiológicos, clínicos e laboratoriais precisos e robustos e em tempo real é fundamental para orientar a tomada de decisões, sobretudo quanto a questões de saúde pública (XU et al., 2020). Logo, exigências de velocidade e eficiência na disseminação dos resultados científicos impõem um desafio adicional ao sistema de comunicação científica (TORRES-SALINAS et al., 2020; LARIVIÈRE; SHU; SUGIMOTO, 2020).

É nesse sentido que o surto ocasionado pelo novo coronavírus tem posto em evidência premissas já conhecidas sobre a relevância do compartilhamento de dados, desenvolvimentos e resultados de pesquisa de forma mais rápida (e aberta) do que a do atual sistema de comunicação científica (LARIVIÈRE; SHU; SUGIMOTO, 2020). Nesta corrida contra o tempo instaurada pela pandemia, a comunidade científica tem reforçado o apelo aos principais publishers e financiadores para implementação de políticas de acesso aberto (open access'), visando incentivar a disponibilização em modo online e sem restrições dos resultados de investigação científica, especialmente aqueles relacionados ao novo coronavírus.

Além disso, as revistas estão reduzindo o tempo de revisão por pares e publicando preprints ${ }^{2}$, com prioridade às publicações relacionadas à COVID-19, prática comum em se tratando da emergência de temas de saúde pública, como também foi o caso do Zika vírus (ARAÚJO et al., 2017). Nesse ínterim, também têm surgido novas plataformas de compartilhamento de pesquisas científicas, como a R\&D Blueprint da OMS (OMS, 2020), responsável por atualizar diariamente informações sobre o novo coronavírus e a CORD-19 (Open Research Dataset Challenge), que compartilha de forma aberta artigos publicados e pre-prints, incluindo estudos anteriores de outros coronavírus que datam da década de 1950 (COLAVIZZA, et al, 2020; BRAINARD, 2020), entre outros. Assim, a resposta por parte dos editores tem sido positiva. Até julho de 2020, mais de 50 editores dos principais periódicos científicos têm disponibilizado gratuitamente os artigos e pesquisas relacionadas à COVID-19 em diferentes plataformas e repositórios como a PubMed Central (PMC) (PMC, 2020).

Concomitantemente, medidas de distanciamento social, restrições de viagens e de mobilidade urbana, desaceleração de negócios e a própria quarentena têm impulsionado a interação pela sociedade em torno de fenômenos ocasionados pela COVID-19 em ambientes, plataformas e ferramentas online, tal qual o Twitter (CHEN; LERMAN; FERRARA, 2020). É a primeira vez na história da humanidade na qual uma pandemia pode ser descrita, debatida e investigada em tempo real e online pela

\footnotetext{
' Open access se refere à disponibilização online, livre e irrestrita de dados e produções científicas (BUDAPESTE, 2002), através de duas estratégias complementares: via verde (green road) referente ao auto-arquivamento em repositórios digitais; e via dourada (golden road) em alusão às publicações em periódicos de acesso aberto (VALERO; CABO; CASTIEL, 2007).

2 Os preprints são versões pré-publicadas de um artigo científico ainda não revisadas por pares (SWAN, 2016).
} 
comunidade científica por meios considerados convencionais (artigos de periódicos), juntamente com mídias sociais (BOETTO et al., 2020).

Iniciativas como as empreendidas por editores e o fortalecimento da interação nas redes sociais em torno de pesquisas científicas vão em direção à chamada Ciência Aberta, termo abrangente que envolve práticas de compartilhamento em acesso aberto, mas que também preconiza maior transparência e valorização da participação de não cientistas e não especialistas na produção e difusão de conhecimento (ALBAGLI; CLINIO; RAYCHTOCH, 2014), constituindo um "novo fazer cientifico" (OLIVEIRA; SILVA, 2016).

Estudos que analisaram e quantificaram a resposta da comunidade científica ante outras emergências internacionais de saúde pública, como o SARS, MERS, H1N1, Ebola e Zika, demonstraram que o número de publicações científicas aumentou imediatamente depois da declaração do surto pela OMS (ZHANG et al., 2020; HAGHANI et al., 2020). No caso da COVID-19, não foi diferente, a declaração oficial da pandemia gerou um crescimento sem precedentes de publicações científicas de diversas áreas do conhecimento, cujo volume se duplica a cada 20 dias (BRAINARD, 2020).

Assim, muitos autores têm apresentado análises sobre o volume crescente das publicações relacionadas à COVID-19 usando indicadores bibliométricos tradicionais, como a dinâmica da publicação, principais países, instituições, autores, revistas, fontes de financiamento, distribuição por áreas do conhecimento, redes de colaboração, entre outros (ZHANG et al., 2020; COLAVIZZA et al., 2020; HAGHANIA et al., 2020; CHAHROUR et al., 2020; LOU et al., 2020; LAKSHAM et al., 2020; ZHOU et al., 2020; ZHAI et al., 2020; MAO et al., 2020; DE FELICE; POLIMENI, 2020). Entretanto, estes artigos não apresentam uma associação entre indicadores bibliométricos e altmétricos sob a perspectiva do acesso aberto, tampouco empreendem uma análise do impacto e difusão destas publicações. Dentro desse contexto, o objetivo do artigo é analisar o impacto do acesso aberto na produção e difusão do conhecimento sobre a COVID-19 nos meios tradicionais de comunicação científica e na web social.

A primeira seção do artigo apresenta os delineamentos metodológicos da pesquisa cuja originalidade consiste na combinação de indicadores bibliométricos e altmétricos, e na comparação entre as publicações compartilhadas em acesso aberto com as de acesso fechado/restrito. Na sequência são discutidos os principais resultados, em termos da dinâmica de publicação (janeiro-abril 2020); indicadores de impacto científico das publicações; redes de cooperação entre instituições, e outros. Finalmente, apontamos as principais considerações, nas quais salientamos que as publicações em acesso aberto permitiram aumentar a difusão e impacto das pesquisas relacionadas à COVID-19, tanto na comunidade acadêmica como na sociedade em geral. Além disso, observamos que redes de cooperação de pesquisa entre instituições científicas e países apresentaram maior índice de publicações em acesso aberto, revelando um fluxo de cooperação institucional mais intenso no enfrentamento da pandemia. 


\section{DELINEAMENTOS METODOLÓGICOS}

Este é um estudo descritivo de abordagem quantitativa, que utiliza tanto indicadores bibliométricos ${ }^{3}$ quanto altmétricos ${ }^{4}$, para identificar o crescimento, difusão e impacto das publicações acadêmicas relacionadas com o novo coronavírus (SARS-CoV-2). Numa perspectiva de complementaridade aos indicadores tradicionais (BAHETI; BHARGAVA, 2017; WILLIAMS, 2017), que permitem analisar a dinâmica das publicações durante determinado período de tempo, é possível, por meio da altmetria, apreender a interação das pessoas na web social em torno de um produto de pesquisa (PIWOWAR, 2013) e avaliar o impacto científico tendo como base atividades e ferramentas online (PRIEM et al., 2010). Parte-se do pressuposto de que a combinação de métodos permite atualizar o conceito de influência científica (BARROS, 2015). Particularmente, as métricas alternativas são importantes para a análise de temas emergentes e de importância global, tal qual a COVID-19, uma vez que dispõem de indicadores quase imediatos de difusão e apropriação da produção científica para além do ambiente acadêmico e dos canais usuais de divulgação (WILLIAMS, 2017; BORREGO, 2014).

Além da combinação de indicadores bibliométricos e altmétricos, foi feita uma comparação entre as publicações académica compartilhadas em acesso aberto com as de acesso fechado/restrito, para entender o impacto do acesso aberto na produção e difusão do conhecimento sobre a COVID-19. As diferentes etapas metodológicas se descrevem a seguir:

a) Identificação de palavras-chave, bases de dados e equações de pesquisa.

Foram utilizadas equações de pesquisa validadas previamente pelo "Observatorio Iberoamericano de la Ciencia, Tecnología y la Sociedad (OCTS)" ", que está acompanhando em tempo real as publicações científicas relacionadas com a COVID-19. O OCTS utiliza a base de dados da PubMed Central (PMC), especializada em artigos da área da Medicina e Ciências da Vida.

Buscando complementar as informações da PubMed com publicações de outras áreas do conhecimento, foi feita uma busca na base de dados multidisciplinar Scopus, cuja equação de pesquisa corresponde à equação utilizada pelo Centro de Informação da Elsevier sobre o novo coronavírus ${ }^{6}$. Uma vez refinadas essas duas equações ${ }^{7}$, foram coletados dados somente de artigos científicos e reviews (todos documentos peerreview). O período da busca foi entre janeiro e abril de 2020. No Quadro 1 são apresentadas as equações de busca utilizadas para a pesquisa.

a) Processamento da informação.

Foi utilizado o software de mineração de dados VantagePoint. Nesta etapa, foram unidos os metadados das duas bases de dados e excluídos os artigos duplicados. Alguns metadados foram complementados através da plataforma Altmetric.com, ferramenta aberta de dados qualitativos e quantitativos sobre o impacto de um

\footnotetext{
3 Bibliometria: é um termo primeiramente proposto por Pritchard (1969) para designar a aplicação de métodos estatísticos e matemáticos para examinar e entender as dinâmicas e produção científica de um determinado campo, mapeando as comunidades acadêmicas, redes de pesquisadores e seu impacto científico através de indicadores como número de citações, autores, instituições, palavras-chave entre outras (OKUBO, 1997).

${ }^{4}$ Altmetria: conjunto de métricas fundamentadas em mídias sociais, softwares de gerenciamento de referência online, enciclopédias colaborativas, blogs e sites de organização de conferências (PRIEM et al, 2010).

5Para mais informações, consultar: <https://observatoriocts.oei.org.ar/2020/03/25/el-radar-del-observatorioctsseguimiento-en-vivo-del-covid-19/>.

${ }^{6}$ Para mais informações, consultar: <https://www.elsevier.com/connect/coronavirus-information-center>.

7 Estas duas equações foram adaptadas para que os resultados da busca fossem relacionados diretamente com à COVID-19 e assim evitar publicações de outros coronavírus.
} 
produto de pesquisa. Para visualização das redes de cooperação e cálculo de suas métricas, foi utilizado o software Gephi;

O número de artigos analisados segundo a modalidade de acesso a nível do artigo (acesso aberto ou fechado) é apresentado na Tabela 1. A modalidade de acesso dos documentos é a nível de artigo, incluindo artigos de periódicos de acesso aberto, acesso fechado e híbridos. Após a fusão das bases e a limpeza de registros com dados duplicados e inconsistentes, o total de artigos ou reviews analisados foi de 6.631 documentos, sendo 4.692 com acesso aberto e 1.939 com acesso fechado.

\section{Quadro 1 - Equações de busca para a identificação de artigos relacionados com a COVID-19.}

\begin{tabular}{|l|l|}
\hline Base de dados & Equação de busca \\
\hline \multirow{5}{*}{ PubMed } & "COVID-19"[TIAB] OR "severe acute respiratory syndrome coronavirus 2" \\
& [Supplementary Concept] OR "severe acute respiratory syndrome coronavirus \\
& 2"[TIAB] OR "2019-nCOV"[TIAB] OR "SARS-CoV-2"[TIAB] OR "2019nCoV"[TIAB] \\
& OR ("Wuhan"[TIAB] AND ("coronavirus"[MeSH Terms] OR \\
& "coronavirus"[TIAB])) AND ("2020/01/01"[PDAT]: "2020/04/30"[PDAT]) \\
\hline \multirow{5}{*}{ Scopus } & TITLE-ABS-KEY ("COVID-19") OR TITLE-ABS-KEY ("2019-nCoV") OR TITLE-ABS- \\
& KEY ("SARS-CoV-2") OR TITLE-ABS-KEY ("Severe Acute Respiratory Syndrome \\
& 2") OR (TITLE-ABS-KEY ("wuhan") AND (TITLE-ABS-KEY (coronavirus) OR TITLE- \\
& ABS-KEY ("Corona virus"))) AND (LIMIT-TO ( DOCTYPE , "ar") OR LIMIT-TO \\
& (DOCTYPE, "re")) AND (LIMIT-TO (PUBYEAR, 2020)) \\
\hline
\end{tabular}

Nota: Período da busca: janeiro-abril de 2020. Data da busca: 15 de maio de 2020.

Fonte: Elaboração própria.

Tabela 1 - Número de artigos relacionados com a COVID-19.

\begin{tabular}{lccc}
\hline $\begin{array}{c}\text { Base de } \\
\text { dados }\end{array}$ & $\begin{array}{r}\text { Acesso } \\
\text { Aberto }\end{array}$ & $\begin{array}{c}\text { Acesso } \\
\text { Fechado }\end{array}$ & Total \\
\hline Scopus & 3.460 & 1.004 & 4.464 \\
PubMed & 3.839 & 2.116 & 5.955 \\
Total & $\mathbf{4 . 6 9 2}$ & $\mathbf{1 . 9 3 9}$ & $\mathbf{6 . 6 3 1}$ \\
\hline
\end{tabular}

Fonte: Elaboração própria a partir de PubMed e Scopus.

b) Identificação e processamento de indicadores da altmetria.

Os identificadores dos artigos selecionados - ID PubMed e DOI (Digital Object Identifier) - foram inseridos na Altmetric.com. A Tabela 2 descreve os dados obtidos. Dos 3.449 artigos de acesso aberto com DOls válidos na plataforma, 3.436 (99,6\%) receberam alguma menção na web social. Do conjunto composto pelos artigos de acesso fechado (1.305 artigos), 1.299 (99,5\%) receberam atenção online. No total, foram mais de um milhão de menções em torno dos artigos de acesso aberto e cerca de 333 mil para os de acesso fechado. 
Tabela 2 - Dados de menções na Plataforma Altmetric

\begin{tabular}{lll}
\hline Indicadores & $\begin{array}{l}\text { Acesso } \\
\text { Aberto }\end{array}$ & Acesso Fechado \\
\hline $\begin{array}{l}\text { No. de artigos válidos no Altmetric } \\
\text { (input) }\end{array}$ & 3.449 & 1.305 \\
$\begin{array}{l}\text { No. de artigos com atenção online } \\
\text { (output) }\end{array}$ & $3.436(99,6 \%)$ & $1.299(99,5 \%)$ \\
Total de menções & & \\
.....Twitter & 1.115 .943 & 333.204 \\
.....Sites de Noticias & 1.063 .013 & 319.557 \\
.....Blog & 42.170 & 9.508 \\
.....Facebook (post públicos) & 4.270 & 1.131 \\
.....Reddit & 3.836 & 1.484 \\
.....Video & 1.494 & 322 \\
.....Wikipedia & 457 & 100 \\
.....Documentos de política & 390 & 56 \\
..... Outros & 192 & 25 \\
Média de menções/artigo & 121 & 21 \\
\hline
\end{tabular}

*Artigos válidos são aqueles que possuem DOI e puderam ser rastreados pela Altmetric.com.

Fonte: Elaboração própria com base em dados da Altmetric.com.

\section{RESULTADOS E DISCUSSÃO}

A Figura 1 apresenta a dinâmica de publicação dos artigos de janeiro a abril de 2020, classificados por sua disponibilidade de acesso. Percebe-se um crescimento significativo de publicação de artigos e revisões sobre a COVID-19, principalmente devido ao grande impacto que a pandemia impõe à população e à ciência. A taxa de crescimento média mensal do período analisado é de $166 \%$. Tal crescimento ocorre tanto em publicações com acesso aberto como em acesso fechado, sendo maior o número de publicações dentro do primeiro conjunto.

Figura 1 - Dinâmica das publicações (artigos e reviews) sobre a COVID-19.

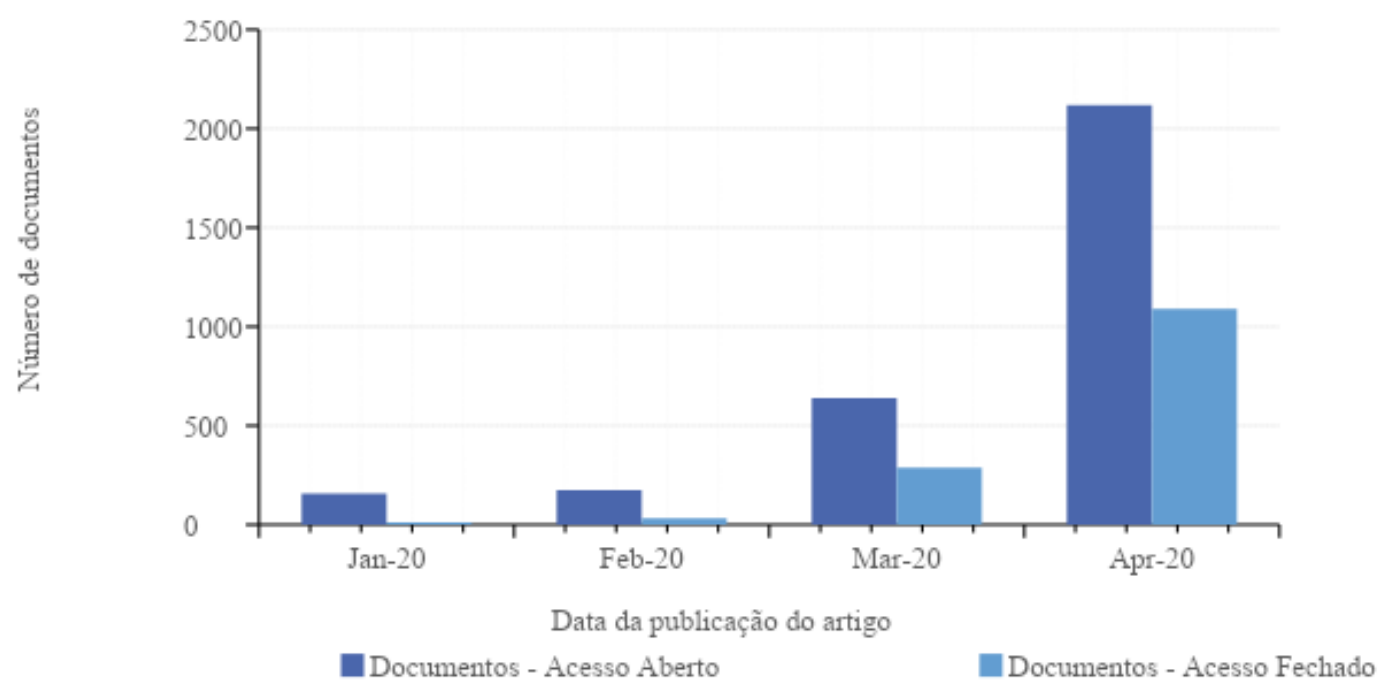

Fonte: Elaboração própria a partir de Scopus e PubMed. 
A Tabela 3 apresenta indicadores de impacto científico das publicações nas duas modalidades (acesso aberto e acesso fechado). Pode-se verificar que ambos os conjuntos de artigos apresentam a maior parte de suas publicações em periódicos de grande relevância científica (com base no indicador "Publications in Q1 Journal Quartile by CiteScore"). Entretanto, os artigos com acesso aberto apresentam um maior impacto da citação ponderada por área do conhecimento (medido pelo "Field Weighted Citation Impact") e uma maior difusão de conhecimento ("Number of Citing Countries") que os artigos com acesso fechado. Especificamente, os artigos de acesso aberto são citados quase 26 vezes mais do que a média de artigos da mesma área do conhecimento e os de acesso fechado são citados quase 10 vezes mais.

Tabela 3 - Indicadores de impacto científico.

\begin{tabular}{lccc}
\hline & $\begin{array}{l}\text { Field-Weighted } \\
\text { Citation Impact }\end{array}$ & $\begin{array}{l}\text { Number of Citing } \\
\text { Countries }^{2}\end{array}$ & $\begin{array}{l}\text { Publications in Q1 Journal } \\
\text { Quartile by CiteScore (\%) }^{3}\end{array}$ \\
\hline COVID-19 - Acesso Aberto & 25,9 & 155 & 63,1 \\
\hline COVID-19 - Acesso Fechado & 9,63 & 139 & 65,4 \\
\hline Notas: & &
\end{tabular}

${ }^{1}$ O Field-Weighted Citation Impact $(\mathrm{FWCl})$ revela como o número de citações recebidas por um conjunto de publicações se compara ao número médio de citações recebidas por todas as outras publicações semelhantes no universo dos dados.

${ }^{2}$ Este indicador mostra a visibilidade geográfica das publicações, a partir do número de países distintos que citaram o conjunto de publicações.

${ }^{3}$ As publicações em quartis de periódicos no SciVal indicam quantas publicações existem nos periódicos classificados nos quartis 1, 2, 3 ou 4 indexados pelo Scopus. No caso, foram utilizadas as métricas do CiteScore para identificar os periódicos correspondentes ao Quartil 1 (Q1), que representa os periódicos melhor classificados na área do conhecimento de cada artigo.

Fonte: Elaboração própria, a partir de dados da plataforma Scival.

A atenção sobre as publicações científicas relacionadas à COVID-19 também se evidencia pelas menções no Twitter, que representam mais de $95 \%$ do total das menções identificadas na web social. Considerando que há uma correlação significativa entre o fluxo de informações no Twitter e os novos casos de COVID-19 (SINGH et al., 2020), as informações dessa mídia social podem ajudar a mapear a propagação e o surto da doença, pois ajudam a entender quanto, onde e como as pessoas estão se comunicando sobre a pandemia. Nesse sentido, a Figura 2 destaca o número de tweets em torno de publicações sobre o tema. 
Figura 2 - Número de tweets em torno de publicações sobre COVID-19, de janeiro a abril (2020).

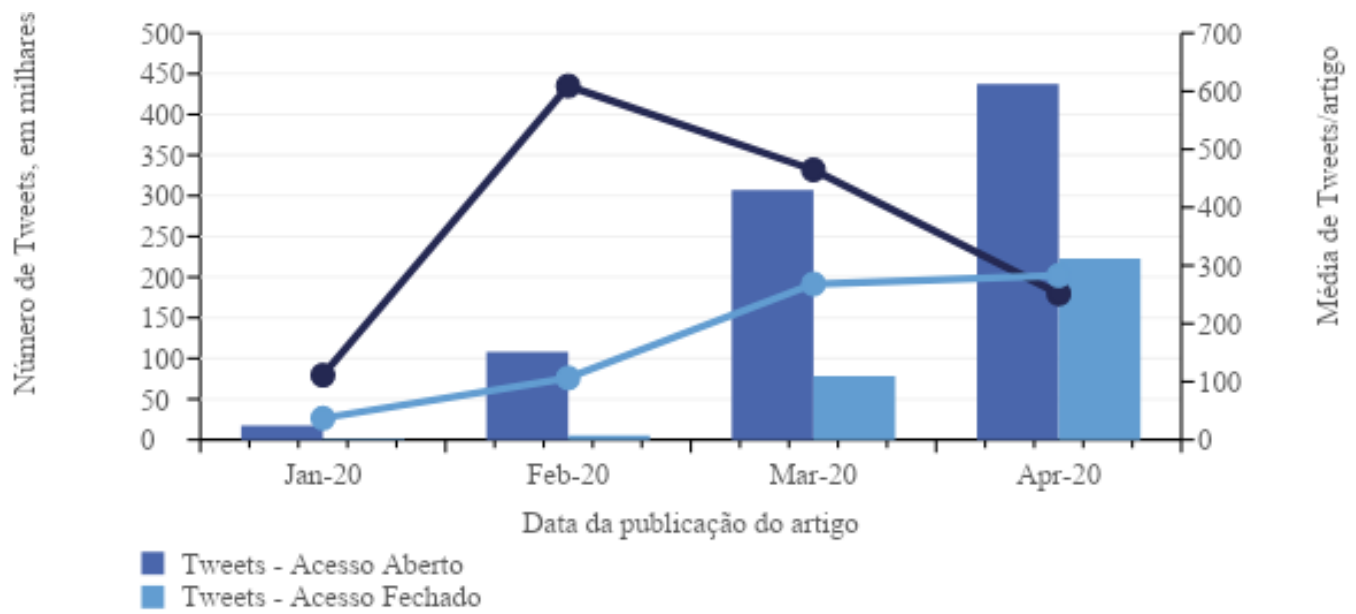

Fonte: Elaboração própria com base em dados disponibilizados pela Altmetric.com.

Os tweets foram crescentes desde janeiro, sendo que abril marcou 437 mil tweets sobre pesquisas em acesso aberto e 222 mil tweets sobre pesquisas em acesso fechado. No entanto, as linhas representadas na Figura 2 normalizam esses números, uma vez que há mais artigos de acesso aberto do que de fechado, o que poderia enviesar a análise, justificando o cálculo da média mensal do número de tweets por artigo. Até março de 2020, as publicações dentro do conjunto de acesso aberto, além de receberem mais menções no Twitter (número absoluto), apresentaram maior média de menção por artigo. Essa configuração se altera em abril, de modo que a média de tweets/artigo acesso fechado passa a ser ligeiramente maior (283 tweets por artigo) do que aqueles de acesso aberto (251 tweets por artigo). Torres-Salinas et al. (2020), trabalhando com uma amostra de 11.686 publicações, obtêm resultados semelhantes, concluindo que publicações de acesso aberto tendem a receber a maior parte da atenção da mídia social.

A Tabela 3 apresenta o Top 10 dos países com maior participação nas publicações e a participação do Brasil. É evidente o esforço dos diferentes países em relação à produção de conhecimento na área, sendo os com maior número de publicações aqueles que tiveram mais casos registrados da COVID-19, até o dia 30 de abril de $2020^{8}$. Os achados de Singh et al., (2020) também evidenciam que a atenção está focada nos países mais afetados pela doença. É importante salientar que, embora o Brasil não esteja dentro do Top 10 países (no contexto temporal da pesquisa), é a nação latinoamericana com maior número de artigos publicados, seguido, respectivamente, pelo México e Colômbia. A despeito de não fornecermos informações qualitativas sobre o conteúdo dos tweets, Kouzy et al. (2020), em seu estudo com uma amostra de 673 tweets, revelam que mais de $80 \%$ dos tweets continham conteúdos fidedignos referentes à pandemia da COVID-19, sendo considerada pequena a parcela de informações erradas ou não verificáveis. 
Tabela 3 - Países com maior participação no número de publicações (Top 10) e a participação do Brasil.

\begin{tabular}{|c|c|c|c|c|c|}
\hline \multirow{2}{*}{$\begin{array}{l}\text { Acesso Aberto } \\
\text { País }\end{array}$} & \multirow[b]{2}{*}{ \#Pub. ${ }^{1}$} & \multirow[b]{2}{*}{$\%$} & \multicolumn{2}{|l|}{ Acesso Fechado } & \multirow[b]{2}{*}{$\%$} \\
\hline & & & País & \#Pub. ${ }^{1}$ & \\
\hline$E_{U} A^{2}$ & 1262 & 28,0 & China $^{2}$ & 520 & 26,8 \\
\hline China $^{2}$ & 1175 & 26,1 & $\mathrm{EUA}^{2}$ & 499 & 25,7 \\
\hline Itália² & 479 & 10,6 & Reino Unido² & 292 & 15,1 \\
\hline Reino Unido ${ }^{2}$ & 401 & 8,9 & Itália² & 163 & 8,4 \\
\hline Canadá & 204 & 4,5 & Índia & 85 & 4,4 \\
\hline França $^{2}$ & 194 & 4,3 & Canada & 54 & 2,8 \\
\hline Alemanha $^{2}$ & 192 & 4,3 & Austrália & 53 & 2,7 \\
\hline Índia & 182 & 4,0 & Irã ${ }^{2}$ & 46 & 2,4 \\
\hline Austrália & 140 & 3,1 & Alemanha² & 45 & 2,3 \\
\hline Espanha $^{2}$ & 132 & 2,9 & França $^{2}$ & 40 & 2,1 \\
\hline Brasil (Posição 16) & 76 & 1,7 & Brasil (Posição 14) & 24 & 1,2 \\
\hline
\end{tabular}

Fonte: Elaboração própria a partir de Scopus e PubMed.

Do ponto de vista de redes sociais, entre as publicações de acesso aberto, os tweets partiram especialmente dos Estados Unidos (16\%), Japão (5\%) e Reino Unido (5\%). Já no conjunto de acesso fechado, dos Estados Unidos (19\%), Reino Unido (7\%) e Espanha (3\%) (Tabela 4).

Tabela 4 - Países com maior participação no número de Tweets em publicações (Top 10).

\begin{tabular}{lll|lll}
\hline Acesso Aberto & & \multicolumn{4}{l}{ Acesso Fechado } \\
País & Tweets & $\%$ & País & Tweets & $\%$ \\
\hline EUA* & 171.881 & 16 & EUA* & 60.57 & 19 \\
& & & & 9 & \\
Japão & 50.789 & 5 & Reino & 22.357 & 7 \\
Reino & 49.778 & 5 & Espanha* & 10.466 & 3 \\
Unido* & & & & & \\
Espanha* & 28.225 & 3 & Japão & 9.998 & 3 \\
Canadá & 20.838 & 2 & Brasil & 7.298 & 2 \\
França* & 17.658 & 2 & Canadá & 6.886 & 2 \\
Austrália & 14.904 & 1 & França* & 4.959 & 2 \\
Brasil & 14.902 & 1 & Austrália & 4.844 & 2 \\
Índia & 14.489 & 1 & México & 3.860 & 1 \\
México & 12.217 & 1 & Índia & 2.909 & 1 \\
\hline * Os 10 países com maiores números de casos de CoVID-19 em 30/04/2020
\end{tabular}

Fonte: Elaboração própria com base em dados disponibilizados pela Altmetric.com. 
O Quadro 2 apresenta um comparativo dos artigos mais mencionados na web social considerando se acesso aberto ou acesso fechado, e o país de filiação de seus autores em uma distinção entre "Brasil" e "Demais países".

\section{Quadro 2 - Artigos com maior atenção online, segundo a modalidade (acesso aberto e acesso fechado).}

\begin{tabular}{|c|c|c|c|c|}
\hline & & $\mathrm{AT}^{1}$ & Artigo & PERIÓDICO \\
\hline \multirow{2}{*}{ 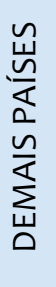 } & $\begin{array}{l}\text { Acesso } \\
\text { Aberto }\end{array}$ & 19.931 & $\begin{array}{l}\text { Projecting the transmission dynamics of SARS- } \\
\text { CoV-2 through the post pandemic period }\end{array}$ & Science \\
\hline & $\begin{array}{l}\text { Acesso } \\
\text { Fechado }\end{array}$ & 11.653 & $\begin{array}{l}\text { Characteristics of and Important Lessons } \\
\text { From the Coronavirus Disease } 2019 \text { (COVID-19) } \\
\text { Outbreak in China }\end{array}$ & $\begin{array}{l}\text { JAMA: Journal of } \\
\text { the American } \\
\text { Medical } \\
\text { Association }\end{array}$ \\
\hline \multirow{2}{*}{ 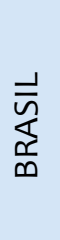 } & $\begin{array}{l}\text { Acesso } \\
\text { Aberto }\end{array}$ & 383 & $\begin{array}{l}\text { Slum Health: Arresting COVID-19 and } \\
\text { Improving Well-Being in Urban Informal } \\
\text { Settlements }\end{array}$ & $\begin{array}{l}\text { Journal of Urban } \\
\text { Health }\end{array}$ \\
\hline & $\begin{array}{l}\text { Acesso } \\
\text { Fechado }\end{array}$ & 28 & $\begin{array}{l}\text { Temperature significantly changes COVID-19 } \\
\text { transmission in (sub) tropical cities of Brazil }\end{array}$ & $\begin{array}{l}\text { Science of the } \\
\text { Total Environment }\end{array}$ \\
\hline
\end{tabular}

${ }^{1}$ O Altmetric Attention Score (AT) é uma medida ponderada de toda a atenção on-line que a Altmetric rastreou para determinado produto de pesquisa. Inclui menções em redes sociais, documentos de políticas públicas, referências na Wikipedia e em sites de notícias, blogs etc. ${ }^{9}$

Fonte: Elaboração própria com bases em dados da Altmetric.com.

As duas publicações com maior atenção online no âmbito global têm em comum a discussão do impacto da COVID-19 no período pós surto. Usando estatísticas de sazonalidade e imunidade, a publicação dentro do conjunto "acesso aberto" mais mencionada na web é de autores da Universidade de Harvard e faz uma projeção da dinâmica de transmissão do SARS-CoV-2 no período posterior à pandemia (KISSLER et al., 2020). Já no contexto do acesso fechado, a publicação em evidência nas redes sociais é de autores do Centro Chinês de Controle e Prevenção de Doenças e discute aprendizados do surto da doença no contexto chinês (WU; MCGOOGAN, 2020).

No cenário brasileiro, Corburn et al. (2020), direcionados à gestão pública, propõem um conjunto de práticas e políticas para diminuir a propagação da COVID-19 em assentamentos informais urbanos no Brasil, considerados menos preparados para enfrentar a pandemia, já que carecem de infraestrutura básica e têm superlotação e restrição de espaço que dificultam ações de distanciamento físico. $\mathrm{O}$ artigo, publicado em acesso aberto, tem seus autores vinculados a várias instituições nacionais e internacionais, sendo a instituição brasileira de afiliação a Universidade Federal de Minas Gerais. No caso da publicação de acesso fechado (de autores da Universidade de Brasília), o destaque é para Prata, Rodrigues e Bermejo (2020), que buscam determinar a relação da temperatura com a infecção por COVID-19 nas capitais brasileiras, focando, portanto, em fatores ecológicos que afetam a transmissão e a sobrevivência do vírus, enquanto o outro artigo brasileiro enfatizou aspectos de natureza socioeconômica, especialmente.

9 Informações disponíveis em: <https://www.altmetric.com/about-our-data/our-sources/.>. 
A colaboração entre instituições e países também se mostra necessária para que o desenvolvimento científico ocorra de forma intensa e acelerada (LEE; BOZEMAN, 2005), principalmente quando a temática da pesquisa é de relevância mundial, como o caso do novo coronavírus. Nessa perspectiva, a Figura 3 mostra a proporção de publicações sem cooperação (publicações com apenas um autor), publicações com cooperação institucional (com dois ou mais autores pertencentes a mesma instituição), publicações com cooperação nacional (com dois ou mais autores pertencentes a diferentes instituições do mesmo país) e publicações com cooperação internacional (com dois ou mais autores pertencentes a instituições de diferentes países). Evidencia-se uma cooperação mais intensa em publicações de acesso aberto, que apresenta uma menor proporção de publicações sem colaboração (com autor único) e maior colaboração a nível nacional e internacional.

Figura 3 - Perfil de colaboração de publicações de acesso aberto e acesso fechado

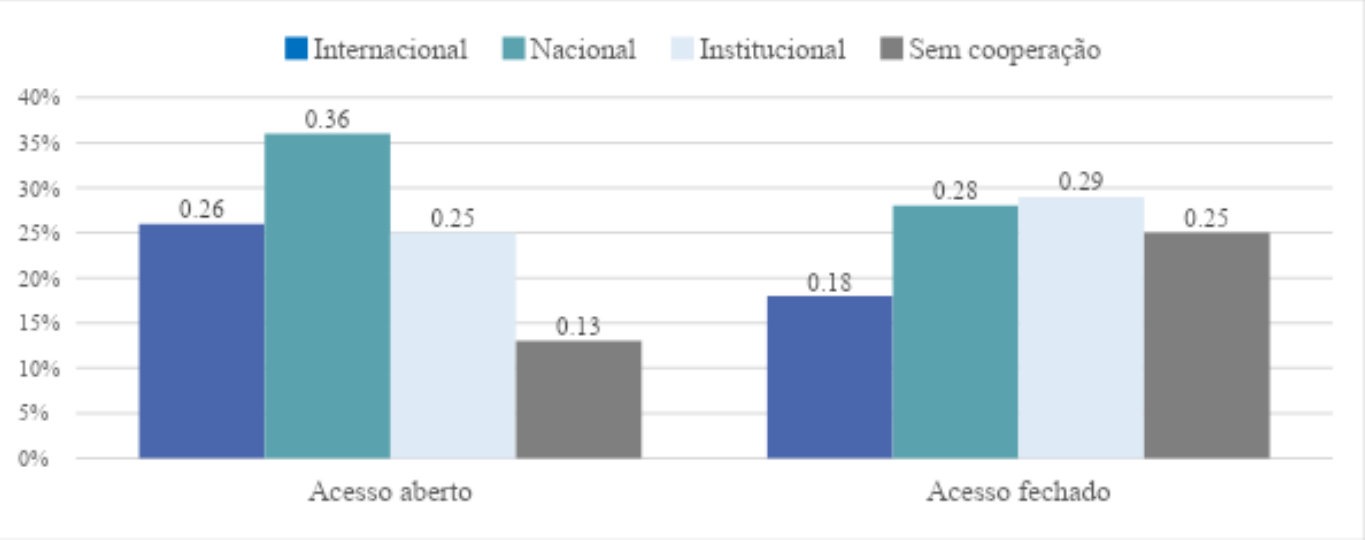

Fonte: Elaboração própria.

As Figuras 4 e 5 mostram as redes de cooperação entre as 20 instituições líderes em publicações e as instituições brasileiras presentes na base de publicações sobre a COVID-19. A Figura 4 trata da rede de cooperação em publicações de acesso aberto e a Figura 5 trata dos dados em publicações de acesso fechado. EUA, China, Hong Kong e Canadá são países que contam com instituições entre as que mais publicam artigos no tema. No caso do Brasil, destacam-se a Fiocruz, USP, UNIFESP e UNICAMP. 
Figura 4 - Rede de Cooperação do top 20 instituições e instituições brasileiras, Acesso Aberto.

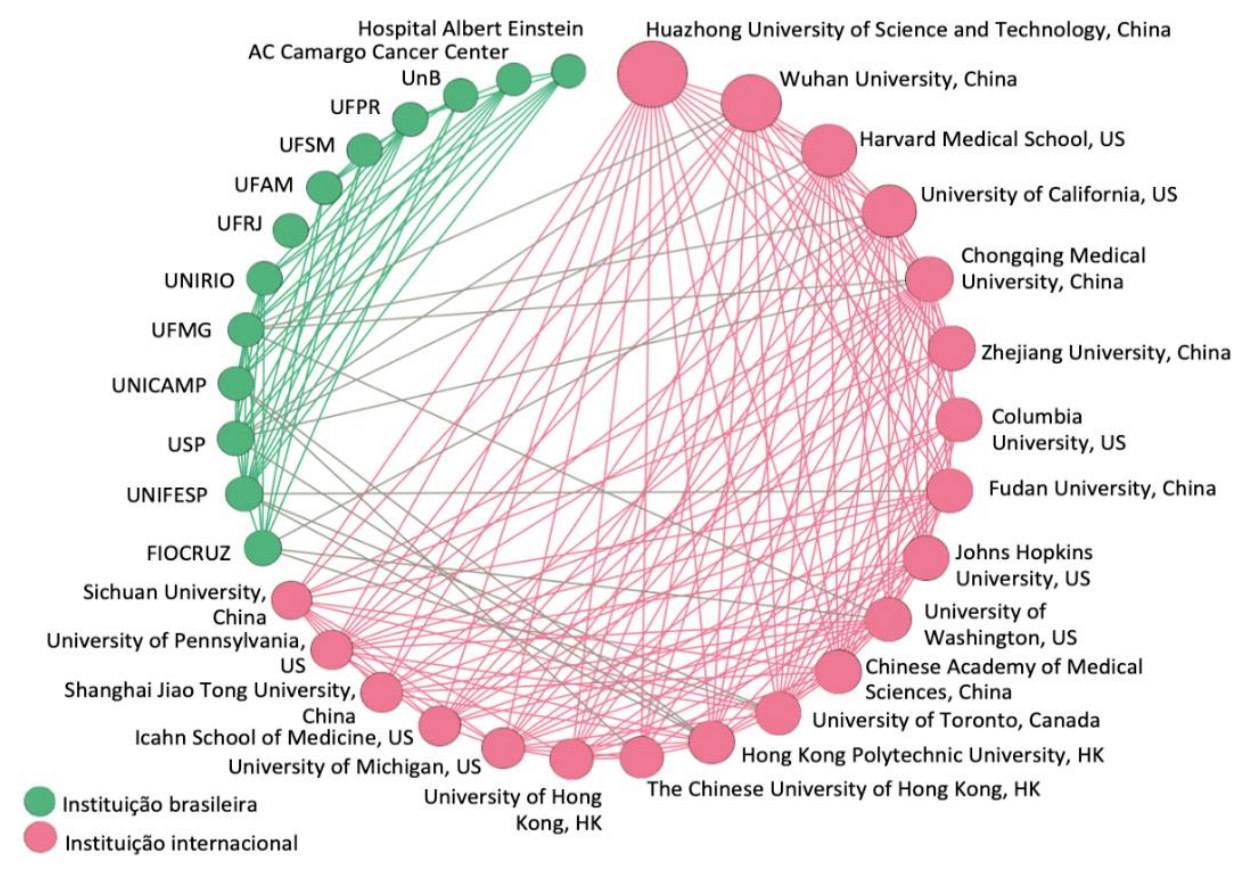

Fonte: Elaboração própria.

Figura 5 - Rede de Cooperação do top 20 instituições e instituições brasileiras, Acesso Fechado

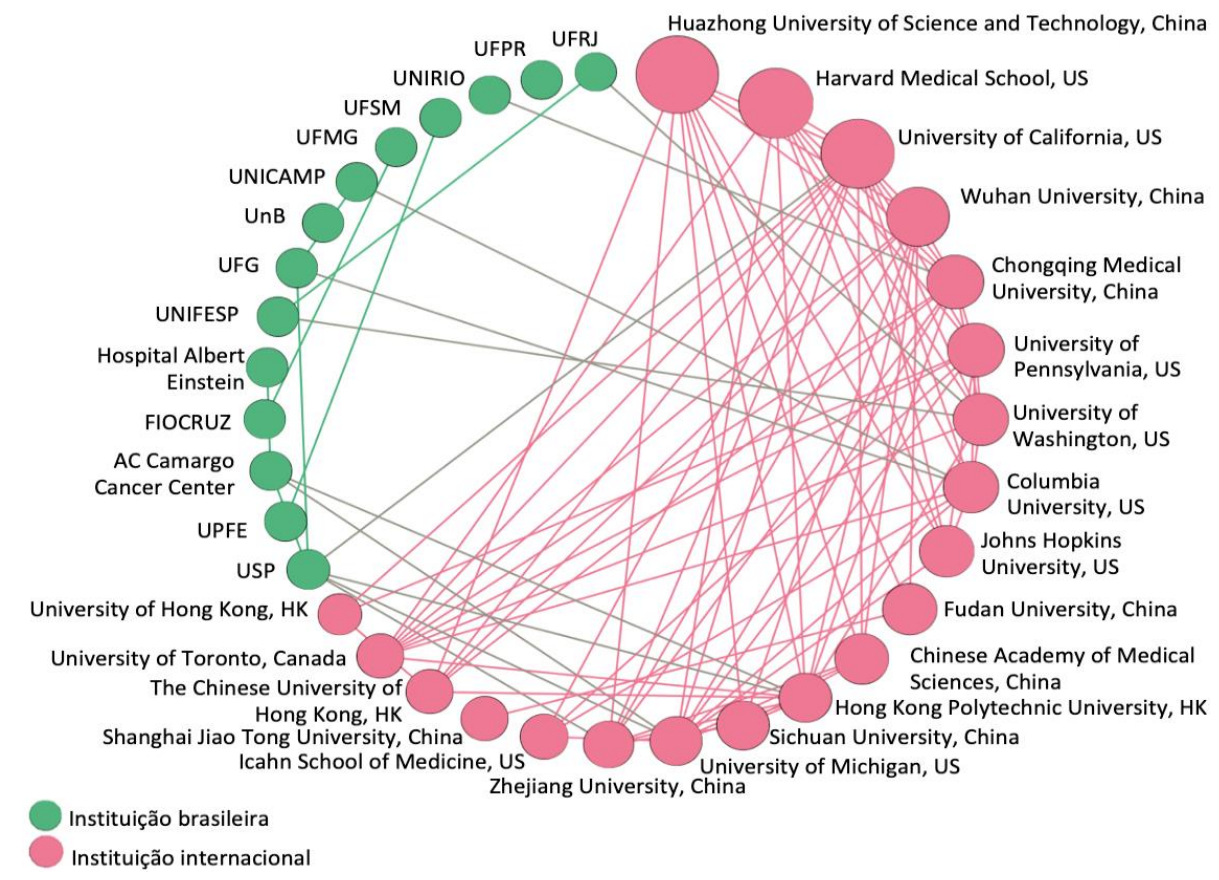

Fonte: Elaboração própria.

As métricas das redes de cooperação (baseadas em WASSERMAN; FAUST, 1994), representadas no Quadro 3, evidenciam que o nível de cooperação é mais intenso quando se trata de artigos em acesso aberto. O grau médio (número de conexões em cada nó) e o grau ponderado médio (ponderado pelo número de artigos que cada instituição possui em cooperação com outra instituição) da rede de cooperação em 
acesso aberto se mostra mais que o dobro dos valores da rede de cooperação em acesso fechado. Já o diâmetro da rede, que indica a maior distância entre duas instituições da rede, revela que a rede de cooperação em acesso aberto é mais próxima e com uma distância de apenas 3 conexões entre as instituições mais distantes da rede. Dentre todas as conexões possíveis de serem realizadas entre as instituições da rede, a de cooperação em acesso aberto possui uma densidade de 0,364 de todas conexões possíveis, enquanto na rede de cooperação em acesso fechado este valor é de 0,164. Também se percebe uma rede estreita de cooperação entre instituições brasileiras que compõem a base de dados, com algumas cooperações pontuais com as instituições que fazem parte do Top 20, em ambas as redes.

Quadro 3 - Métricas das redes de cooperação.

\begin{tabular}{l|c|c|c|c|}
\hline & Grau Médio & $\begin{array}{c}\text { Grau Ponderado } \\
\text { Médio }\end{array}$ & $\begin{array}{c}\text { Diâmetro da } \\
\text { Rede }\end{array}$ & $\begin{array}{c}\text { Densidade do } \\
\text { Grafo }\end{array}$ \\
\hline $\begin{array}{l}\text { Acesso Aberto } \\
\text { (Fig. 4) }\end{array}$ & 11,636 & 23,273 & 3 & 0,364 \\
$\begin{array}{l}\text { Acesso Fechado } \\
\text { (Fig. 5) }\end{array}$ & 5,412 & 10,824 & 5 & 0,164 \\
\hline
\end{tabular}

Fonte: Elaboração própria a partir do software Gephi.

Finalmente, na Tabela 5, estão listadas as principais revistas científicas que publicaram artigos ou reviews relacionados com a COVID-19, tanto em acesso aberto como em acesso fechado.

Tabela 5 - Revistas científicas com maior número de artigos sobre a COVID-19 disponibilizados em acesso aberto e em acesso fechado.

\begin{tabular}{|c|c|c|c|c|c|}
\hline \multicolumn{3}{|c|}{ Artigos em Acesso Aberto } & \multicolumn{3}{|c|}{ Artigos em Acesso Fechado } \\
\hline Revista & SJR2019 ${ }^{1}$ & \#Pub. ${ }^{2}$ & Revista & SJR2019' & \#Pub. ${ }^{2}$ \\
\hline $\begin{array}{l}\text { Clinical Infectious } \\
\text { Diseases }\end{array}$ & 4,226 & 99 & $\begin{array}{l}\text { BMJ - British Medical } \\
\text { Journal }\end{array}$ & 1,247 & 206 \\
\hline $\begin{array}{l}\text { Journal of Medical } \\
\text { Virology }\end{array}$ & 0,855 & 91 & $\begin{array}{l}\text { Journal of the } \\
\text { American Medical } \\
\text { Association }\end{array}$ & 5,913 & 56 \\
\hline $\begin{array}{l}\text { Infection Control \& } \\
\text { Hospital Epidemiology }\end{array}$ & 1,555 & 67 & $\begin{array}{l}\text { Journal of Medical } \\
\text { Virology }\end{array}$ & 0,855 & 45 \\
\hline The Lancet & 14,554 & 65 & Head \& Neck & 1,16 & 41 \\
\hline $\begin{array}{l}\text { Annals of Internal } \\
\text { Medicine }\end{array}$ & 4,738 & 57 & $\begin{array}{l}\text { Chinese Journal of } \\
\text { Epidemiology }\end{array}$ & - & 40 \\
\hline $\begin{array}{l}\text { International Journal } \\
\text { of Infectious Diseases }\end{array}$ & 1,437 & 57 & $\begin{array}{l}\text { Chinese Journal of } \\
\text { Tuberculosis and } \\
\text { Respiratory Diseases }\end{array}$ & - & 34 \\
\hline $\begin{array}{l}\text { Anesthesia \& } \\
\text { Analgesia }\end{array}$ & 1,41 & 47 & $\begin{array}{l}\text { New England } \\
\text { Journal of Medicine }\end{array}$ & 18,291 & 27 \\
\hline $\begin{array}{l}\text { Emerging Infectious } \\
\text { Diseases }\end{array}$ & 2,72 & 45 & $\begin{array}{l}\text { Chinese journal of } \\
\text { preventive medicine }\end{array}$ & 0,228 & 26 \\
\hline $\begin{array}{l}\text { European Heart } \\
\text { Journal }\end{array}$ & 5,883 & 38 & Circulation & 7,446 & 25 \\
\hline $\begin{array}{l}\text { International Journal } \\
\text { of Environmental } \\
\text { Research and Public } \\
\text { Health }\end{array}$ & 0,739 & 37 & $\begin{array}{l}\text { Indian Journal of } \\
\text { Community Health }\end{array}$ & 0,132 & 25 \\
\hline
\end{tabular}

Fonte: Elaboração própria. 
Diante da pandemia do novo coronavírus, as principais revistas da área da medicina e ciências da vida decidiram que a maioria de suas publicações ficariam em acesso aberto para facilitar a difusão dos resultados da pesquisa nesta área. Assim, revistas que tradicionalmente cobram uma assinatura para ter acesso a seu conteúdo, têm disponibilizado plataformas exclusivas com informações sobre a Covid-19. Este é o caso de revistas como The Lancet, Infection Control \& Hospital Epidemiology, Annal of Internal Medicine, entre outras.

Contudo, outras editoras de revistas científicas têm adotado um modelo, um tanto quanto controverso, de acesso aberto híbrido, no qual uma parte de suas publicações são disponibilizadas através do sistema comercial por assinatura, e outra parte em acesso aberto mediante a cobrança de uma taxa de publicação dos próprios autores (SWAN, 2016). Por esta razão, é possível que uma mesma revista tenha artigos tanto em acesso aberto como em acesso restrito.

Em relação às publicações feitas por pesquisadores brasileiros, grande parte se concentra em revistas brasileiras que disponibilizam artigos tanto em acesso aberto como em acesso fechado (Vide Tabela 6).

Tabela 6 - Revistas científicas com publicações de autores brasileiros ${ }^{3}$, disponibilizados em acesso aberto e acesso fechado.

\begin{tabular}{|c|c|c|c|c|c|}
\hline \multicolumn{3}{|c|}{ Artigos em Acesso Aberto } & \multicolumn{3}{|c|}{ Artigos em Acesso Fechado } \\
\hline Revista & SJR2019' & \#Pub². & Revista & SJR2019' & \#Pub². \\
\hline $\begin{array}{l}\text { Cadernos de Saúde } \\
\text { Pública }\end{array}$ & 0,565 & 5 & $\begin{array}{l}\text { Science of the Total } \\
\text { Environment }\end{array}$ & 1,661 & 4 \\
\hline $\begin{array}{l}\text { Revista Epidemiologia e } \\
\text { Serviços de Saúde }\end{array}$ & 0,489 & 4 & $\begin{array}{l}\text { American Journal of } \\
\text { Otolaryngology }\end{array}$ & 0,614 & 2 \\
\hline $\begin{array}{l}\text { Journal of Medical } \\
\text { Virology }\end{array}$ & 0,855 & 4 & $\begin{array}{l}\text { Asian Pacific Journal of } \\
\text { Tropical Medicine }\end{array}$ & 0,539 & 1 \\
\hline $\begin{array}{l}\text { Journal of the Brazilian } \\
\text { Society of Tropical } \\
\text { Medicine }\end{array}$ & 0,531 & 4 & Chemotherapy & 0,488 & 1 \\
\hline $\begin{array}{ll}\text { JBRA } & \text { Assisted } \\
\text { Reproduction } & \end{array}$ & - & 3 & Drug Discovery Today & 1,896 & 1 \\
\hline $\begin{array}{l}\text { Brazilian Journal of } \\
\text { Psychiatry }\end{array}$ & 0,708 & 2 & $\begin{array}{l}\text { International Brazilian } \\
\text { Journal of Urology }\end{array}$ & 0,471 & 1 \\
\hline Clinics & 0,419 & 2 & $\begin{array}{l}\text { Jornal Brasileiro de } \\
\text { Pneumologia }\end{array}$ & 0,478 & 1 \\
\hline $\begin{array}{l}\text { Hematology, Transfusion } \\
\text { and Cell Therapy }\end{array}$ & - & 2 & $\begin{array}{l}\text { BMJ - British Medical } \\
\text { Journal }\end{array}$ & 1,247 & 1 \\
\hline Jornal de Pediatria & 0,63 & 2 & Head \& Neck & 1,16 & 1 \\
\hline $\begin{array}{l}\text { Pan American Journal of } \\
\text { Public Health }\end{array}$ & 0,41 & 2 & Medical Education & 1,711 & 1 \\
\hline
\end{tabular}

${ }^{1}$ SJR (SCImago Journal Rank) consiste no cálculo do prestígio das citações recebidas pela revista e envolve a proporção do valor das citações dependendo do campo, qualidade e reputação da revista que cita. O valor médio de SJR para todas revistas indexadas no Scival é 1.

2 \#Pub. refere-se ao número de publicações de cada revista.

3 Os artigos desta tabela correspondem a publicações com ao menos um autor com filiação institucional brasileira.

Fonte: Elaboração própria.

Dentre as principais revistas nas quais publicam autores brasileiros, se destacam Cadernos de Saúde Pública, Revista Epidemiologia e Serviços de Saúde, Journal of Medical Virology e Journal of the Brazilian Society of Tropical Medicine, que, juntas, 
correspondem a 22\% de todas publicações em acesso aberto. Já em relação aos artigos de acesso fechado de autores brasileiros, $25 \%$ foram publicados pelas revistas Science of the Total Environment e American Journal of Otolaryngology.

As revistas de destaque em publicações de artigos sobre COVID-19 apresentam, em sua maioria, uma relevância científica alta, conforme também pode ser verificado na Tabela 1, apresentada anteriormente. No entanto, as publicações de autores brasileiros sobre a COVID-19, independente de sua acessibilidade, concentram-se em revistas consideradas de menor prestígio (SJR2019<1).

\section{CONSIDERAÇÕES FINAIS}

Práticas de difusão de publicações em acesso aberto e redes de colaboração são ambas facetas de um modelo de Ciência que tem ganhado visibilidade junto aos quadros epistemológicos e institucionais vigentes denominado como Ciência Aberta (Open Science). Os resultados apresentados neste artigo evidenciam que o volume das publicações científicas relacionadas à COVID-19 triplicou na média mensal entre janeiro e abril de 2020. Nesse crescimento, destacam-se as publicações na modalidade acesso aberto, o que pode ser explicado pelo fato das principais revistas, periódicos e bases de dados terem adotado, ao menos temporariamente, políticas de Acesso Aberto, disponibilizando de modo eletrônico e gratuito pesquisas e artigos relacionados com o novo coronavírus. Estas iniciativas estão alinhadas com o que, anteriormente, foi preconizado pela OMS para enfrentamento da epidemia do Zika Virus, e quando se estabeleceu que todas as publicações e pesquisas relacionadas com uma emergência de saúde pública deveriam ser compartilhadas de forma aberta (OMS, 2015). Assim, este estudo reforça a importância do acesso aberto na comunicação científica, tendo como objetivo respostas científicas colaborativas, rápidas e oportunas para entender e tratar tais emergências.

Os países que têm maior número de publicações são aqueles que tiveram mais casos registrados da COVID-19 até o dia 30 de abril de 2020. No cenário latino-americano, o Brasil é o país com maior número de artigos publicados, tendo a Fiocruz um papel proeminente nesse sentido. No âmbito global, se sobressaem universidades chinesas da região de Wuhan e universidades americanas.

A evolução e a disseminação da produção científica vieram acompanhadas de menções sobre o tema também nas redes sociais, principalmente no Twitter. Os tweets em torno de publicações sobre a COVID-19 foram crescentes desde janeiro. No geral, com exceção ao mês de abril, as publicações científicas dentro do conjunto acesso aberto, além de receberem mais menções no Twitter, apresentaram maior média de menção por artigo. Os tweets partiram especialmente dos Estados Unidos e do Reino Unido.

Além disso, mostramos que a produção de conhecimento sobre a COVID-19 impulsionou a formação de redes de cooperação entre instituições científicas e países, mais intensamente no conjunto das publicações em acesso aberto. Percebe-se uma rede estreita de cooperação entre instituições brasileiras que compõem a base de dados, com algumas cooperações pontuais com instituições internacionais que fazem parte do Top 20.

Diante dos achados, a principal contribuição do artigo parte da escolha metodológica, na qual a combinação entre indicadores bibliométricos e altmétricos permitiu não somente caracterizar a evolução e difusão da produção científica sobre a COVID-19, mas gerou evidências para compreender a relação entre a pandemia provocada pelo novo coronavírus e a interação da sociedade em torno dos produtos de pesquisa. $O$ uso de redes sociais como o Twitter possibilitou que os artigos científicos em acesso 
aberto e fechado fossem difundidos além da comunidade acadêmica, permitindo que a sociedade em geral tivesse acesso a informações relevantes e validadas cientificamente sobre a COVID-19.

No cenário estudado, concluímos que o modelo de comunicação científica online, livre e sem restrições, tal qual o preconizado pelo acesso aberto, e amplamente adotado pelas principais editoras científicas no contexto da pandemia, mostrou-se fundamental para a o fortalecimento de redes de colaboração de pesquisa e para a produção e difusão do conhecimento sobre este tema emergente e de interesse mundial. A corrida científica instaurada pela pandemia da COVID-19 permitiu visualizamos de uma forma robusta os impactos desse modelo, preconizando a importância sustenta-lo no longoprazo.

Sugere-se, para estudos futuros, uma análise mais aprofundada das redes de cooperação e difusão de conhecimento, buscando entender, qualitativamente, os tipos de relações desenvolvidas em função da disponibilidade de dados das pesquisas sobre a COVID-19.

\section{AGRADECIMENTOS}

Algumas instituições foram essenciais para o desenvolvimento deste artigo. Especificamente, os autores gostariam de agradecer: à CAPES (Coordenação de Aperfeiçoamento de Pessoal de Nível Superior), pela possibilidade de acesso aos banco de dados de publicações; ao GEOPI (Laboratório de Estudos sobre Organização da Pesquisa e da Inovação - DPCT/IG/UNICAMP), pelo acesso ao software Vantage point; ao Altmetric.com, pelo acesso às análises da plataforma; e ao DPCT/IG pela formação e união deste grupo de autores.

\section{REFERÊNCIAS}

ALBAGLI, S.; CLINIO, A.; RAYCHTOCK, S. Ciência aberta: correntes interpretativas e tipos de ação. Liinc em Revista, v. 10, n. 2, 2014.

ARAÚJO, K. Mendonça de et al. A produção científica sobre zika em periódicos de acesso aberto. RECIIS, Rio de Janeiro, v. 11, p. 1-8, nov. 2017. Suplemento.

BAHETI, A. D.; BHARGAVA, P. Altmetrics: a measure of social attention toward scientific research. Current Problems in Diagnostic Radiology, v. 46, n. 6, p. 391-392, 2017.

BARROS, M. Altmetrics: métricas alternativas de impacto científico com base em redes sociais. Perspectivas em Ciência da Informação, v. 20, n. 2, p. 19-37, 2015.

BOETTO, E. et al. Using altmetrics for detecting impactful research in quasi-zero-day time-windows: the case of COVID-19. 2020.

BORREGO, Á. Altmétricas para la evaluación de la investigación y el análisis de necesidades de información. Profesional de la Información, v. 23, n. 4, p. 352-358, 2014.

BRAINARD, J. Scientists are drowning in COVID-19 papers: can new tools keep them afloat?. Science, 2020. 
BUDAPESTE. Budapeste open access initiative. Disponível em:

https://www.budapestopenaccessinitiative.org/. Acesso em 23 de maio de 2020.

CHAHROUR, M. et al. A Bibliometric analysis of COVID-19 Research activity: a call for increased output. Cureus, v. 2, n. p. 1-8, 2020.

CHEN, E.; LERMAN, K.; FERRARA, E. Covid-19: The first public coronavirus twitter dataset. 2020.

COLAVIZZA, G. et al. A scientometric overview of CORD-19. bioRxiv, p. 2020.04.20.046144, 2020.

CORBURN, J. et al. Slum health: arresting COVID-19 and improving well-being in urban informal settlements. Journal of Urban Health, p. 1-10, 20-?.

DE FELICE, F.; POLIMENI, A. Coronavirus disease (COVID-19): a machine learning bibliometric analysis. In vivo (Athens, Greece), v. 34, n. 3, p. 1613-1617, 2020.

GORBALENYA, A. E. Severe acute respiratory syndrome-related coronavirus-the species and its viruses, a statement of the coronavirus study group. BioRxiv. 2020. Doi:10.1101/2020.02.07.937862. 2020.

HAGHANI, M. et al. The scientific literature on Coronaviruses, COVID-19 and its associated safety-related research dimensions: a scientometric analysis and scoping review. Safety Science, v. 129, n. May, 2020.

KISSLER, S. M. et al. Projecting the transmission dynamics of SARS-CoV-2 through the postpandemic period. Science, v. 368, n. 6493, p. 860-868, 2020.

KOUZY, R. et al. Coronavirus goes viral: quantifying the COVID-19 misinformation epidemic on Twitter. Cureus, v. 12, n. 3, 2020.

LAKSHAM, S. et al. Mapping the research output on coronavirus: a scientometric study. Gedrag en Organisatie, v. 33, n. 2, p. 163-186, 2020.

LARIVIÈRE, V.; SHU, F.; SUGIMOTO, C. The Coronavirus (COVID-19) outbreak highlights serious deficiencies in scholarly communication. LSE Impact of Social Sciences. Disponível em: https://blogs.

Ise.ac.uk/impactofsocialsciences/2020/03/05/the-coronavirus-covid-19-outbreakhighlights-serious-deficiencies-in-scholarly-communication. Acesso em: 25 de abril de 2020.

LEE, S.; BOZEMAN, B. The impact of research collaboration on scientific productivity. Social Studies of Science, v. 35, p. 673-702, 2005.

LOU, J. et al. Coronavirus disease 2019: A bibliometric analysis and review. European Review for Medical and Pharmacological Sciences, v. 24, n. 6, p. 3411-3421, 2020.

MAO, X. et al. The status and trends of coronavirus research: a global bibliometric and visualized analysis. Medicine, v. 99, n. 22, p. e20137, 2020.

OKUBO, Y. Bibliometric indicators and analysis of research systems: methods and examples. [S.I.]: OECD ilibrary, Publishing Systems, 1997. 
OLIVEIRA, A. C. S. de; SILVA, Edilene Maria da. Ciência aberta: dimensões para um novo fazer científico. Informação \& Informação, v. 21, n. 2, p. 5-39, 2016.

ORGANIZAÇÃO MUNDIAL DA SAÚDE - OMS. R\&D Blueprint and COVID-19. Disponível em: https://www.who.int/teams/blueprint/covid-19. Acesso em: 11 jul. 2020.

ORGANIZAÇÃO MUNDIAL DA SAÚDE - OMS. Developing global norms for sharing data and results during public health emergencies, 2015. Disponível em:

https://www.who.int/medicines/ebola-treatment/blueprint_phe_data-shareresults/en/. Acesso em: 03 out. 2020.

ORGANIZAÇÃO MUNDIAL DA SAÚDE - OMS et al. Who director-general's opening remarks at the media briefing on COVID-19. Geneva, Switzerland, 2020.

PIWOWAR, H. Value all research products. Nature, v. 493, p. 159, 2013.

PRATA, D. N.; RODRIGUES, W.; BERMEJO, P. H. Temperature significantly changes COVID-19 transmission in (sub) tropical cities of Brazil. Science of the Total Environment, p. 138862, 2020.

PRIEM, J. et al. Altmetrics: a manifesto. 2010. Disponível em: http://altmetrics.org/manifesto. Acesso em: 11 mar. 2020.

PRITCHARD, A. Statistical bibliography or bibliometrics?. Journal of Documentation, v. 24, n. 4, p. 348-349, 1969.

PUBMED CENTRAL. Public Health Emergency COVID-19 Initiative. 2020. Disponível em: https://www.ncbi.nlm.nih.gov/pmc/about/covid-

19/?fbclid=IwAR05xNYVrbSE6eBy3wCiVS9Y_R1zISo4nOrprspcg1MFoNIGURatmHQex Mk. Acesso em: 11 jul. 2020.

SINGH, L. et al. A first look at COVID-19 information and misinformation sharing on Twitter. arXiv preprint arXiv:2003.13907,2020.

SWAN, A. Diretrizes para as políticas de desenvolvimento e promoção do acesso aberto. [S.I.]: UNESCO Brasil, IBICT, 2016. Disponível em:

https://unesdoc.unesco.org/ark:/48223/pfooo0246018. Acesso em 13 abr. 2020.

TORRES-SALINAS, D.; ROBINSON-GARCIA, N.; CASTILLO-VALDIVIESO, P. A. Open access and Altmetrics in the pandemic age: forescast analysis on COVID-19 related literature. BioRxiv, 2020.

VALERO, J. S.; CABO. J. V.; CASTIEL, L. D. A iniciativa Open Access no acesso à informação técnico-científica nas Ciências da Saúde. 2007.

WASSERMAN, S.; FAUST, K. Social network analysis: methods and applications. Cambridge Univ. Press, Cambridge, U.K. 1994.

WILLIAMS, A. E. Altmetrics: an overview and evaluation. Online Information Review, v. 41, n. 3, p. 311-317, 2017.

WU, Z.; MCGOOGAN, J. M. Characteristics of and important lessons from the coronavirus disease 2019 (COVID-19) outbreak in China: summary of a report of 72314 cases from the Chinese Center for Disease Control and Prevention. Jama, v. 323, n. 13, p. 1239-1242, 2020. 
$\mathrm{XU}$, B. et al. Open access epidemiological data from the COVID-19 outbreak. The Lancet Infectious Diseases, v. 20, n. 5, 534. 2020.

ZHAI, F. et al. Research progress of coronavirus based on bibliometric analysis. International Journal of Environmental Research and Public Health, v. 17, n. 11, p. 1-15, 2020.

ZHANG, L. et al. How scientific research reacts to international public health emergencies: a global analysis of response patterns. Scientometrics, n. 0123456789 , 2020.

ZHOU, Y.; CHEN, L. Twenty-year span of global coronavirus research trends: a bibliometric analysis. International Journal of Environmental Research and Public Health, v. 17, n. 9, 2020. 